\title{
Gestión participativa en el desarrollo de las instituciones públicas
}

\author{
Mg. Elia Aracely Córdova Calle \\ eliacc22@hotmail.com
}

\author{
Dr. José Manuel Delgado Bardales \\ manueldelgado1506@hotmail.com
}

\section{RESUMEN}

En el trabajo se realizó con el propósito de conocer y proponer mejoras en la gestión participativa en beneficio de las instituciones gubernamentales. El principal elemento estudiado es la gestión participativa, donde el trabajo consensuado y articulado ayuda a cumplir las metas institucionales y al desarrollo de las mismas. La investigación es descriptiva, con enfoque cualitativo y la revisión sistemática de artículos nacionales e internacionales referidas a la variable gestión participativa que luego fueron analizadas. Se concluye que casi el 100\% de los estudios relacionan la influencia de la gestión participativa en el desarrollo de las instituciones públicas. Estas están basadas en políticas que tiene que ver con procesos desde la planificación, organización, dirección y control. Incluso, las instituciones inician el proceso desde la parte interna y los que deben fomentar la articulación externa con buenas políticas de comunicación para lograr la participación activa y compromisos de cada uno, con miras a generar desarrollo o llegar a objetivos comunes. Para lograr la articulación de los diferentes actores depende mucho de la decisión política de los gobernantes o decisores de las instituciones, con vocación de servicio para generar desarrollo social.

Palabras clave: Gestión, participación, desarrollo y articulación. 


\title{
Participatory management in the development of public institutions
}

\begin{abstract}
The work was carried out with the purpose of knowing and proposing improvements in participatory management for the benefit of government institutions. The main element studied is participatory management, where consensual and articulated work helps to meet institutional goals and their development. The research is descriptive, with a qualitative approach and a systematic review of national and international articles referring to the participatory management variable, which were later analyzed. It is concluded that almost $100 \%$ of the studies relate the influence of participatory management in the development of public institutions. These are based on policies that have to do with processes from planning, organization, direction and control. Even the institutions start the process from the internal part and those that must promote external articulation with good communication policies to achieve the active participation and commitments of each, with a view to generating development or reaching common objectives. To achieve the articulation of the different actors depends a lot on the political decision of the governors or decision-makers of the institutions, with a vocation of service to generate social development.
\end{abstract}

Keywords: Management, participation, development and articulation.

Artículo recibido: 03 nov. 2020 Aceptado para publicación: 07 dic. 2020 Correspondencia: eliacc22@hotmail.com, Conflictos de Interés: Ninguna que declarar 


\section{INTRODUCCIÓN}

Los países pertenecientes a la Unión Europea, experimentaron en los últimos 22 años en sus instituciones públicas, un crecimiento hasta $1.3 \%$, muy por debajo del potencial que realmente tienen para aportar al desarrollo social. Estas instituciones públicas en la actualidad no tienen el rendimiento adecuado respecto al cumplimiento de metas de manera eficaz y eficiente con miras a brindar buenos servicios públicos a los ciudadanos y generar desarrollo. Esto se debe a los débiles procesos de gestión participativa en las instituciones públicas en lo que concierne a procesos de Planificación, organización, dirección y control para logra una buena articulación de actores involucrados, tomando decisiones consensuadas de acuerdo a los objetivos de las instituciones públicas (Bonnal, Cenerini, Ciparissi y Rossi, 2013, p. 20).

En el Perú, las instituciones públicas albergan casi un $73 \%$ de fuerza laboral, considerado como uno de los países Latinoamericanos con mayor índice en el 2013 (Consorcio de Investigación Económica Social [CIES], 2016, p. 3). Las instituciones públicas brindan servicios para disminuir brechas y satisfacer demandas de las grandes mayorías. Sin embargo, la mayoría de instituciones no tienen vocación de servicio reflejando indiferencia por colaborar con los ciudadanos y establecer una mayor participación de los mismos en la elaboración de los instrumentos de gestión, involucrando incluso a todos los colaboradores de la institución para tener mejores resultados (Salgado, Flores y Guevara, 2017).

La región San Martín, ha tenido en los últimos años un buen dinamismo económico con un crecimiento de $4 \%$ y una productividad de 8 mil a 14 mil en términos reales entre el 2007 y 2016, todo esto gracias al aporte de las instituciones públicas (Banco Central de Reserva del Perú [BCRP], 2017, p. 11). Para que las instituciones públicas mejoren llevan a cabo la gestión participativa, conocido como un espacio de colaboración y participación de manera articulada de todos los actores involucrados con una buena comunicación en las instituciones dentro de un marco de colaboración para lograr el desarrollo de los ciudadanos. Sin embargo, esto no se realiza a cabalidad debido a la escasa mano de obra calificada en la administración de las instituciones públicas, haciendo que no haya participación social en la elaboración de políticas públicas y los diferentes programas y proyectos dirigidos a la población, sobre todo para lograr una mejor calidad del gasto, conjugando el interés de éstas con el bien común y colectivo en todos los niveles de gobierno.

Dentro de ello formulamos el siguiente problema

¿Cómo es la gestión participativa en el desarrollo de las instituciones del estado? 
Y como objetivo tenemos:

Conocer y proponer mejoras en la gestión participativa, en el desarrollo de las instituciones públicas.

El propósito del trabajo investigativo, es conocer las características de la gestión participativa y cómo funciona en todas sus dimensiones en las instituciones del estado; en torno a ello, proponer mejoras en el desarrollo de las mismas.

Como justificaciones tenemos las siguientes:

La justificación por conveniencia se enmarca en que las conclusiones de las revisiones bibliográficas, serán de gran utilidad para la implementación de políticas dirigidas a solucionar problemas de articulación interna de gestión y coadyuvar al mejor desempeño y desarrollo de las instituciones públicas.

En la parte social, las conclusiones a través del desarrollo de las instituciones públicas, ayudará a mejorar la calidad de vida de los ciudadanos en el ámbito de la región, dinamizando el desarrollo económico en general con la participación de todos los involucrados.

También, aportará teóricamente respecto a las teorías utilizadas en el trabajo investigativo respecto a la variable de gestión participativa, que será precedente para otras investigaciones en el marco del tema de estudio.

A su vez, tiene implicancia práctica en lo que se refiere a resolver la baja gestión participación en las instituciones públicas, aplicando políticas por las autoridades y directivos correspondientes.

Incluso, a nivel metodológico, se justifica debido a que el diseño descriptivo, la técnica de revisión sistemática con investigaciones científicas y el instrumento, serán usados para investigaciones posteriores con métodos similares.

\section{MATERIAL Y MÉTODOS}

Los materiales utilizados en el trabajo investigativo fue laptop, cuadros de análisis con todos los elementos necesarios para realizar los análisis correspondientes. El método es deductivo, que se partió de hechos generales de resultados de diferentes autores, para aterrizar en hechos particulares y conclusiones especificas respecto al tema.

A su vez, la investigación fue aplicada, con la utilización de teorías establecidas referente al tema investigativo para respaldar la investigación. Como muestra se usaron más de 9 autores en materia de análisis. A su vez, la investigación es de tipo de investigación es cualitativa; como técnica utilizó la revisión sistemática respecto a la variable de gestión participativa, 
como instrumento la guía de análisis sistemática; basándose en estudios de artículos de revistas indexadas, sintetizando resultados y conclusiones identificando la problemática aplicable a muchas realidades.

Los datos se recolectaron mediante la bibliografía de los artículos descritos en los resultados, basados en la variable de gestión participativa en las instituciones gubernamentales. Los artículos fueron a nivel internacional y nacional, accediendo al texto completo de los mismos de los últimos 5 años.

Posteriormente, se realizó el análisis de cada investigación plasmada en la guía de revisión mediante cuadros sistemáticos; los mismos fueron analizados mediante los resultados de cada uno. Después de ello, se realizó la discusión de las conclusiones de cada estudio primario, analizando las discrepancias y similitudes de cada uno, y llegando a conclusiones analíticas con las evidencias encontradas en referencia al objetivo planteado en la investigación; ello sirvió de referencia para llegar a conclusiones finales.

Respecto a la ética, a nivel internacional no se recurrió la utilización de animales ya que no amerita. Se citó a cada uno de los autores comprendidos en la investigación, utilizando la norma internacional APA.

\section{RESULTADOS.}

Referente a los resultados, se presenta en diferentes cuadros de análisis mediante tablas del 1 al 9, con los autores respectivos, con los elementos importantes de ubicación, fuente, metodología, resultados y conclusiones de la siguiente manera: 
Tabla 01:

Datos de publicación de autor 01 .

\begin{tabular}{|c|c|c|c|c|c|c|}
\hline 1. Autor & Año & \multicolumn{3}{|c|}{ Nombre de la Investigación } & Revista donde se ubica la Publicación & Volumen y Número \\
\hline $\begin{array}{c}\text { Salgado, S. Flores, M. } \\
\text { Guevara, M. }\end{array}$ & 2017 & Gestión partici & $\begin{array}{l}\text { tiva para mejorar li } \\
\text { urbana: La Hacie }\end{array}$ & $\begin{array}{l}\text { s condiciones de accesibilidad } \\
\text { da, Puebla. }\end{array}$ & $\begin{array}{l}\text {.redalyc.org/pdf/2033/20335091 } \\
\text { 8026.pdf } \\
\text { León, México }\end{array}$ & $\begin{array}{c}\text { Revista } \\
\text { Nova Scientia, vol. 9, núm. } \\
\text { 18, 2017, pp. 568-587 }\end{array}$ \\
\hline \multicolumn{7}{|c|}{ CONTENIDO } \\
\hline $\begin{array}{l}\text { Tipo y Diseño de } \\
\text { Investigación }\end{array}$ & $\begin{array}{c}\text { Población y } \\
\text { Muestra }\end{array}$ & $\begin{array}{c}\text { Técnica e } \\
\text { instrumentos }\end{array}$ & Ética & Resultados & \multicolumn{2}{|l|}{ Conclusiones } \\
\hline Descriptiva & $\begin{array}{c}\text { Revisión } \\
\text { documentaria y } \\
\text { bibliográfica }\end{array}$ & $\begin{array}{l}\text { Ficha de } \\
\text { recolección de } \\
\text { datos de } \\
\text { Publicaciones }\end{array}$ & $\begin{array}{l}\text { Código de ética } \\
\text { en investigación }\end{array}$ & $\begin{array}{l}\text { La articulación y compromiso de todos los } \\
\text { actores ayudó a desarrollar el proceso de la } \\
\text { gestión participativa. Cada uno expone sus } \\
\text { preocupaciones y problemas para luego ser } \\
\text { consensuados y actuar de acuerdo a sus } \\
\text { funciones. }\end{array}$ & \multicolumn{2}{|c|}{$\begin{array}{l}\text { En los procesos de gestión participativa, y compromiso debe } \\
\text { existir una colaboración de todos los involucrados, desde los } \\
\text { administrativos y la misma población, sumándose otras } \\
\text { instituciones u organizaciones para generar desarrollo y } \\
\text { solucionar los problemas territoriales. }\end{array}$} \\
\hline
\end{tabular}

Fuente: Elaboración propia

Tabla 02

Datos de publicación de autor 02

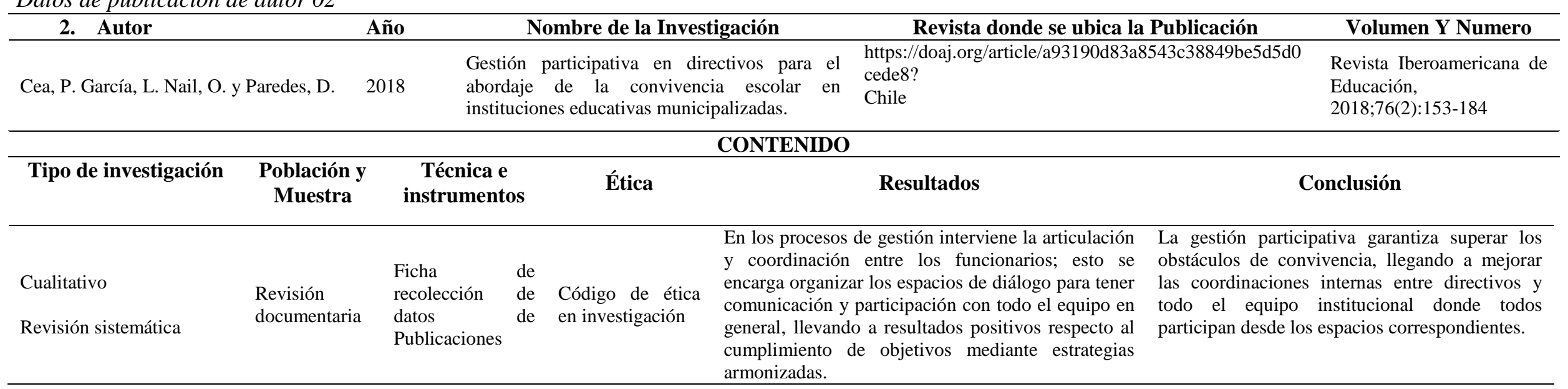

Fuente: Elaboración propia. 


\section{Tabla 03}

Datos de publicación de autor 03.

\begin{tabular}{|c|c|c|c|c|c|c|c|c|}
\hline \multirow{2}{*}{\multicolumn{2}{|c|}{$\begin{array}{l}\text { 3. Autor. } \\
\text { Ramírez, N., Calderón, B., y Milián, M. }\end{array}$}} & \multirow{2}{*}{$\begin{array}{l}\text { Año } \\
2017\end{array}$} & \multicolumn{3}{|c|}{ Título } & \multicolumn{2}{|c|}{ Ubicación de la revista } & Volumen y número. \\
\hline & & & $\begin{array}{l}\text { Gestión pa } \\
\text { estrategias d } \\
\text { histórico de }\end{array}$ & $\begin{array}{l}\text { cipativa en la el } \\
\text { revitalización urbana } \\
\text { n José, Puebla. }\end{array}$ & $\begin{array}{l}\text { oración de } \\
\text { ara el barrio }\end{array}$ & $\begin{array}{l}\text { http://dx.doi.org/10.21640/ns.v9i1 } \\
\text { México, España }\end{array}$ & 680 & $\begin{array}{l}\text { Revista } \quad \text { Nova } \\
\text { scientia vol.9 n.18 León } 20 \\
17\end{array}$ \\
\hline \multicolumn{9}{|c|}{$\begin{array}{ll}\text { CONTENIDO } \\
\end{array}$} \\
\hline Tipo de investigación & $\begin{array}{c}\text { Población y } \\
\text { Muestra }\end{array}$ & & $\begin{array}{l}\text { nica e } \\
\text { Imentos }\end{array}$ & Ética & & Resultados & & onclusión \\
\hline Descriptivo. & $\begin{array}{c}\text { Revisión } \\
\text { documentaria }\end{array}$ & & $\begin{array}{l}\text { e recolección } \\
\text { datos de } \\
\text { licaciones }\end{array}$ & $\begin{array}{l}\text { Código de ética en } \\
\text { investigación }\end{array}$ & $\begin{array}{r}\text { Como r } \\
\text { participat } \\
\text { miembros } \\
\text { integral de } \\
\text { mejoramien } \\
\text { disponibles } \\
\text { parte socia }\end{array}$ & $\begin{array}{l}\text { esultado de las intervenciones } \\
\text { vas y articuladas entre todos los } \\
\text { sirvió de base para la propuesta } \\
\text { revitalización urbana con miras al } \\
\text { to que recupere espacios turísticos } \\
\text { históricos, muy importantes en la } \\
\text {, económica, urbana y ambiental. }\end{array}$ & $\begin{array}{l}\text { Una } \\
\text { la } \\
\text { com } \\
\text { los ac }\end{array}$ & $\begin{array}{l}\text { ortante en las instituciones es } \\
\text { iva, ayuda a fortalecer ideas } \\
\text { jetivos en conjunto con todos } \\
\text { garantiza la integración social } \\
\text { vida de una población. }\end{array}$ \\
\hline
\end{tabular}

\section{Fuente: Elaboración propia}

Tabla 04

Dataos de publicación de autor 04

4. Autor Año $\quad$ Título $\quad$ Ubicación de la revista
Assunção S., Acevedo, B., y $2016 \quad \begin{aligned} & \text { La obra martiana en la gestión participativa } \\ & \text { ambiental para el desarrollo sostenible }\end{aligned}$
$\begin{aligned} & \text { Rodríguez, G.//scielo.sld.cu/scielo.php?script=sci_arttext\&pid=S2218- } \\ & \text { 36202016000100023\&lng=en\&tlng=en\#? Cien Fuegos, Cuba }\end{aligned}$

\section{Volumen y número}

Revista Universidad

sociedad vol.8 no.1 Cienfuegos, Cuba

\section{CONTENIDO}

\begin{tabular}{|c|c|c|c|c|c|}
\hline $\begin{array}{l}\text { Tipo y Diseño de } \\
\text { Investigación }\end{array}$ & $\begin{array}{c}\text { Población y } \\
\text { Muestra }\end{array}$ & Instrumentos & Ética & Resultados & Conclusiones \\
\hline Descriptiva, básica. & $\begin{array}{l}\text { Revisión } \\
\text { documentaria }\end{array}$ & $\begin{array}{l}\text { Evaluación de su estado } \\
\text { general y para } \\
\text { determinar y clasificar la } \\
\text { filtración glomerular }\end{array}$ & $\begin{array}{l}\text { Consentimiento } \\
\text { informado. }\end{array}$ & $\begin{array}{l}\text { La obra martiana se basó en un proceso de concientización } \\
\text { de la población, con la preparación de los autores para } \\
\text { generar cambio, fortaleciendo valores, y asumiendo } \\
\text { compromisos con la participación de todos los involucrados } \\
\text { en la prevención de los problemas ambientales locales. }\end{array}$ & $\begin{array}{l}\text { La gestión participativa se fortalece mediante } \\
\text { objetivos comunes de los involucrados en general. En } \\
\text { el caso de la obra martiniana, articula la participación } \\
\text { de los jóvenes en actividades de cuidado ambiental y } \\
\text { social. }\end{array}$ \\
\hline
\end{tabular}

Fuente: Elaboración propia. 
Tabla 05

Datos de publicación de autor 05

\begin{tabular}{|c|c|c|c|c|c|c|c|c|}
\hline \multirow{2}{*}{\multicolumn{2}{|c|}{$\begin{array}{l}\text { 5. Autor } \\
\begin{array}{l}\text { Marín, F., Riquett, M., Pinto, M. y } \\
\text { otros }\end{array}\end{array}$}} & \multirow{2}{*}{$\begin{array}{l}\text { Año } \\
2017\end{array}$} & \multicolumn{3}{|c|}{ Nombre de la Investigación } & \multirow{2}{*}{\multicolumn{2}{|c|}{$\begin{array}{c}\text { Revista donde se ubica la Publicación } \\
\text { https://www.redalyc.org/pdf/310/31053180015.pdf } \\
\text { Maracaibo, Venezuela }\end{array}$}} & \multirow{2}{*}{$\begin{array}{l}\text { Volumen Y Numero } \\
\text { Revista Opción, vol. 33, } \\
\text { núm. 82, 2017, pp. 344-365 } \\
\text { Universidad del Zulia. }\end{array}$} \\
\hline & & & $\begin{array}{l}\text { Gestió } \\
\text { contex } \\
\text { en esc }\end{array}$ & $\begin{array}{l}\text { participativa y ca } \\
\text { del plan de mejo } \\
\text { las colombianas. }\end{array}$ & $\begin{array}{l}\text { ducativa en el } \\
\text { to institucional }\end{array}$ & & & \\
\hline \multicolumn{9}{|c|}{ CONTENIDO } \\
\hline Tipo de investigación & $\begin{array}{c}\text { Población y } \\
\text { Muestra }\end{array}$ & \multicolumn{2}{|c|}{$\begin{array}{c}\text { Técnica e } \\
\text { instrumento }\end{array}$} & Ética & & esultados & \multicolumn{2}{|c|}{ Conclusiones } \\
\hline Cualitativa y empírica & $\begin{array}{l}\text { Actores claves } \\
\text { del proyecto } \\
\text { elegidos por } \\
\text { conveniencia. }\end{array}$ & \multicolumn{2}{|c|}{$\begin{array}{l}\text { Se aplicaron } \\
\text { entrevistas, } \\
\text { observación y } \\
\text { análisis } \\
\text { documental }\end{array}$} & $\begin{array}{l}\text { Consentimiento } \\
\text { informado. }\end{array}$ & $\begin{array}{l}\text { Como resultad } \\
\text { en el desarrollo } \\
\text { normas existen } \\
\text { de participacic } \\
\text { escolar y la to } \\
\text { positivos. }\end{array}$ & $\begin{array}{l}\text { senta las brechas que existen } \\
\text { lan de mejoramiento con las } \\
\text { demás, la cultura estratégica } \\
\text { yudó a mejorar la gestión } \\
\text { le decisiones con resultados }\end{array}$ & \multicolumn{2}{|c|}{$\begin{array}{l}\text { La gestión participativa forma parte de la gestión } \\
\text { política, donde existe participación de los ciudadanos y } \\
\text { los paradigmas de la mayoría. El compartimento de } \\
\text { concepciones hace que cada involucrado contribuya a } \\
\text { resolver problemas comunes. }\end{array}$} \\
\hline
\end{tabular}

Fuente: Elaboración propia

Tabla 06

Datos de publicación de autor 06

\begin{tabular}{|c|c|c|c|c|c|c|}
\hline 6. & Autor & Año & \multicolumn{2}{|c|}{ Nombre de la Investigación } & Revista donde se ubica la Publicación & Volumen Y Numero \\
\hline \multicolumn{2}{|c|}{ Ramírez, N., Arana, N., y Guevara, M. } & 2019 & \multicolumn{2}{|c|}{$\begin{array}{l}\text { Participative management model for humanizing } \\
\text { public spaces. Analco neighborhood }\end{array}$} & $\begin{array}{c}\text { o://eds.a.ebscohost.com/eds/pdfviewer/pdfview } \\
\text { sid=ee4c9d1b-321d-49d0-a0f9- } \\
\text { ff24a668e79c\%40sessionmgr4008 } \\
\text { Puebla, México. }\end{array}$ & $\begin{array}{l}\text { Revista Bitácora Urbano } \\
\text { Territorial, } 29(1): 43-52\end{array}$ \\
\hline \multicolumn{7}{|c|}{ CONTENIDO } \\
\hline $\begin{array}{c}\text { Tipo de } \\
\text { investigación }\end{array}$ & $\begin{array}{l}\text { Población y } \\
\text { Muestra }\end{array}$ & & cnica e instrumentos & Ética & Resultados & Conclusión \\
\hline $\begin{array}{l}\text { Investigación acción, } \\
\text { cualitativa con } \\
\text { enfoques sucesivos. }\end{array}$ & $\begin{array}{l}\text { Revisión } \\
\text { documentaria }\end{array}$ & $\begin{array}{l}\text { Ficha d } \\
\text { observac }\end{array}$ & $\begin{array}{l}\text { ecolección de datos y guía de } \\
\text { n. }\end{array}$ & $\begin{array}{l}\text { Código de ética en } \\
\text { investigación. }\end{array}$ & $\begin{array}{l}\text { La gestión participativa ayudó a lograr a } \\
\text { fortalecer el manejo de espacios públicos } \\
\text { con contenido histórico, cultural y } \\
\text { paisajístico, articulando acciones con } \\
\text { todos los ciudadanos e instituciones. }\end{array}$ & $\begin{array}{l}\text { La gestión participativa tiene procesos de } \\
\text { apoyo y colaboración de todos, donde } \\
\text { convergen necesidades y objetivos comunes } \\
\text { para lograr el bienestar social. }\end{array}$ \\
\hline
\end{tabular}

Fuente: Elaboración propia

Ciencia Latina Revista Científica Multidisciplinar, Ciudad de México, México.

ISSN 2707-2207 / ISSN 2707-2215 (en línea), julio-diciembre, 2020, Volumen 4, Número 2. https://doi.org/10.37811/cl_rcm.v4i2.160 p.1358 
Tabla 07

Datos de publicación de autor 07

\begin{tabular}{|c|c|c|c|c|c|c|c|c|c|c|}
\hline \multirow[b]{2}{*}{ Lora, P. y Rocha, D. } & & Año & \multicolumn{3}{|c|}{ Nombre de la Investigación } & \multicolumn{2}{|l|}{ Revista donde se ubica la Publicación } & \multicolumn{3}{|c|}{ Volumen Y Numero } \\
\hline & & 2016 & $\begin{array}{l}\text { Promoción de } \\
\text { utilización de } \\
\text { gestión del con }\end{array}$ & $\begin{array}{l}\text { a innovación social a t } \\
\text { metodologías participa } \\
\text { cimiento. }\end{array}$ & $\begin{array}{l}\text { avés de la } \\
\text { ivas en la }\end{array}$ & $\begin{array}{l}\text { http://dx.doi.org/10.19052/ed.3513 } \\
\text { Bogotá }\end{array}$ & & $\begin{array}{l}\text { Revista, } \\
\text { Desarrollo, } \\
178.2016 .\end{array}$ & $\begin{array}{l}\text { Equidad } \\
\text { (25), }\end{array}$ & $\begin{array}{r}\& \\
159- \\
\end{array}$ \\
\hline \multicolumn{11}{|c|}{ CONTENIDO } \\
\hline Metodología & $\begin{array}{l}\text { Población y } \\
\text { Muestra }\end{array}$ & & rumentos & Ética & & Resultados & & lusiones & & \\
\hline $\begin{array}{l}\text { Investigación acción, } \\
\text { participativa. Descriptiva }\end{array}$ & $\begin{array}{l}\text { Muestra } \\
\text { seleccionada } \\
\text { al azahar. }\end{array}$ & & sis documental & $\begin{array}{l}\text { Código de ética en } \\
\text { investigación }\end{array}$ & $\begin{array}{l}\text { Las metod } \\
\text { la gestión } \\
\text { transforma } \\
\text { los diagnó } \\
\text { y esfuerzo }\end{array}$ & $\begin{array}{l}\text { gías participativas están relacionadas con } \\
\text { cial. Todo este proceso, favorece a la } \\
\text { n social. Con la participación de todos en } \\
\text { os, conllevando a integrar conocimientos } \\
\text { todos para lograr objetivos comunes. }\end{array}$ & $\begin{array}{l}\text { El di } \\
\text { pode } \\
\text { un ce } \\
\text { sus c } \\
\text { para } \\
\text { atañe }\end{array}$ & $\begin{array}{l}\text { n elemento im } \\
\text { acciones y neg } \\
\text { ese proceso, } \\
\text { os, experienci } \\
\text { problemas so }\end{array}$ & $\begin{array}{l}\text { mportante } 1 \\
\text { gociacione } \\
\text { todos apo } \\
\text { ias y accic } \\
\text { ociales que }\end{array}$ & $\begin{array}{l}\text { para } \\
\text { es en } \\
\text { ortan } \\
\text { ones } \\
\text { e les }\end{array}$ \\
\hline
\end{tabular}

\section{Tabla 08}

Datos de publicación de autor 08

\begin{tabular}{|c|c|c|c|c|c|}
\hline 8. Autor & & Año & Título & Ubicación de revista & Volumen y número \\
\hline Cayotopa, C. & & $\begin{array}{l}\text { Modelo de ges } \\
\text { integrada y } \\
\text { fortalecimiento de }\end{array}$ & $\begin{array}{l}\text { stión turística municipal, } \\
\text { participativa, para el } \\
\text { la oferta turística cultural. }\end{array}$ & $\begin{array}{l}\text { http://revistas.ucv.edu.pe/index.php/UCV- } \\
\text { HACER/issue/view/194 } \\
\text { Chiclayo, Perú }\end{array}$ & $\begin{array}{l}\text { Revista UCV - Hacer. Vol. } \\
7 \text { Núm. } 3 \text { (2018) }\end{array}$ \\
\hline \multicolumn{6}{|c|}{ CONTENIDO } \\
\hline Metodología & $\begin{array}{c}\text { Población y } \\
\text { Muestra }\end{array}$ & $\begin{array}{c}\text { Técnicas e } \\
\text { instrumentos }\end{array}$ & Ética & Resultados & Conclusiones \\
\hline Cuantitativa & Encuestas & $\begin{array}{l}\text { Actores involucrados de } \\
\text { la comunidad elegidos al } \\
\text { azahar. }\end{array}$ & $\begin{array}{ll} & \text { La ges } \\
& \text { Zaña, } \\
& \text { que se } \\
\text { Código de ética } & \text { particip } \\
\text { en investigación. } & \end{array}$ & $\begin{array}{cl}\text { ión turística en el distrito de } & \text { El protag } \\
\text { enen dificultades y problemas, } & \text { sociales, } \\
\text { resuelven mediante procesos } & \text { participat } \\
\text { tivos de todos los pobladores. } & \text { sobre la } \\
& \text { mecanism } \\
& \text { municipal } \\
& \text { las metas } \\
\end{array}$ & $\begin{array}{l}\text { de los actores involucrados y } \\
\text { que exista una articulación } \\
\text { de todos se involucran y deciden } \\
\text { ón de los recursos, planteando un } \\
\text { ución por procesos liderado por la } \\
\text { presarios y ciudadanos para lograr } \\
\text { on turística. }\end{array}$ \\
\hline
\end{tabular}

Fuente: Elaboración propia 
Tabla 09

Datos de publicación del autor 09

\begin{tabular}{|c|c|c|c|c|c|}
\hline 9. $\quad$ Autor & Año & \multicolumn{3}{|r|}{ Ubicación de revista } & Volumen y número \\
\hline Garrido, M., y Hernández, A. & 2014 & \multicolumn{2}{|c|}{$\begin{array}{l}\text { El patrimonio cultural: Una propuesta de gestion } \\
\text { participative. }\end{array}$} & $\begin{array}{l}\text { http://dehesa.unex.es/handle/10662/4509. } \\
\text { España. }\end{array}$ & $\begin{array}{l}\text { Revista, Tejuelo, no } \\
19 \text { (2014), pág. 62- } \\
75 .\end{array}$ \\
\hline \multicolumn{6}{|c|}{ CONTENIDO } \\
\hline Metodología & $\begin{array}{c}\text { Población y } \\
\text { Muestra }\end{array}$ & $\begin{array}{c}\text { Técnica e } \\
\text { instrumentos }\end{array}$ & Ética & Resultados & Conclusiones \\
\hline Descriptivo & $\begin{array}{l}\text { Pobladores } \\
\text { de la } \\
\text { comunidad. }\end{array}$ & $\begin{array}{ll}\text { Ficha } & \text { de } \\
\text { recolección } & \text { de } \\
\text { datos } & \end{array}$ & $\begin{array}{l}\text { Código de ética } \\
\text { en investigación. }\end{array}$ & $\begin{array}{ll}\text { Hubo participación y colaboración de los } & \text { La participación socia } \\
\text { ciudadanos en la propuesta; sin embargo, } & \text { planificación, seguin } \\
\text { faltó formalidad en el proceso. Respecto a } & \text { pacto, para tener en cl } \\
\text { la gestión participativa, estuvo presente } & \text { y apuntar a un solo se } \\
\text { desde un inicio, con un papel activo y de } & \text { en las comunidades. } \\
\text { involucramiento de todos. } & \end{array}$ & $\begin{array}{l}\text { es importante desde la } \\
\text { iento, negociación y } \\
\text { o los objetivos de todos } \\
\text { tido de desarrollo social }\end{array}$ \\
\hline
\end{tabular}

Fuente: Elaboración propia 


\section{Análisis de revisiones bibliográficas}

Según la revisión realizada se tiene los siguientes resultados de los autores consultados:

En este sentido, Salgado, S. Flores, M. y Guevara, M. (2017), tiene como resultados que la articulación y compromiso de todos los actores ayudó a desarrollar el proceso de la gestión participativa. Cada uno expone sus preocupaciones y problemas para luego ser consensuados y actuar de acuerdo a sus funciones. Por su parte Cea, P. García, L. Nail, O. y Paredes, D. (2018), tiene como resultados que, la articulación y participación son herramientas claves en la colaboración de procesos de gestión, donde impera la comunicación en todos los niveles y el compromiso de todos los actores involucrados para lograr objetivos consensuados.

Sin embargo, Ramírez, N., Calderón, B., y Milián, M. (2017), obtuvo del resultado, que las intervenciones en modo participativo de los ciudadanos, ayudó en la formulación de una propuesta integral de mejoramiento urbana sostenible. Esto se dio con la articulación de todos los actores públicos y privados con los que se logró consolidar la recuperación de espacios importantes con contenido histórico, económico, ambiental, cultural, urbanístico y social. Así mismo, Assunção S., Acevedo, B., y Rodríguez, G. (2016), tuvo como resultados la utilización de un instrumento de participación activa de los pobladores y autoridades e instituciones privadas, con pensamiento Martiniano para asumir responsabilidades dirigidas a cuidar el medio ambiente.

Por su parte, Marín, F., Riquett, M., Pinto, M., Romero, S., y Paredes, A. (2017), muestra en sus resultados que la puesta en marcha de la propuesta de gestión participativa, teniendo en contra la parte normativa de manera restringida. Sien embargo, el compromiso de todos ayudó a tener resultados positivos. Ramírez, N., Arana, N., y Guevara, M., (2019), muestra que la participación e involucramiento de todos, ayudó a mejorar la gestión para lograr recuperar los espacios importantes como patrimonios culturales, históricos y paisajísticos.

A su vez, Lora, P. y Rocha, D. (2016), muestra que, la metodología de participación activa, está enlazada a la gestión social, ayudando a mejorar diagnósticos y soluciones a la problemática que aqueja a todos, realizando una transformación social con la integración de conocimientos. Cayotopa, C. (2018), advierte que la gestión turística del distrito, tuvo una serie de dificultades que se resolvieron mediante la participación de la comunidad, teniendo en cuenta los recursos turísticos disponibles. Incluso, Garrido, M., y Hernández, A. (2014), muestra que existe una relación positiva respecto a la articulación y participación de los ciudadanos, involucrándose de manera colaborativa con la propuesta de trabajo planteada; 
pero a su vez, falto los procesos de formalización de las instituciones y organizaciones del lugar.

La gestión participativa debe integrar procesos desde la planificación en horizontes, la parte organizativa, negociación y pacto con todos los involucrados, con la participación activa, comunicación y compromisos para generar desarrollo.

\section{DISCUSIÓN}

La revisión sistemática de los 9 artículos científicos, sobre gestión participativa en el desarrollo de las instituciones públicas, corresponde a diferentes bases de datos, de autores con investigaciones descriptivas.

Después de análisis de artículos de cada cuadro respectivo, se aprecia que, del total de artículos, el 55\% pertenecen a México, España y Reino Unido y el 45\% son de Chile, Perú y Bogotá. Casi todas las investigaciones aplicaron el análisis documental y descriptivos.

En ese sentido, Salgado, S. Flores, M. y Guevara, M., concluye que los procesos de gestión participativa y compromiso debe existir una colaboración de todos los involucrados, desde los administrativos y la misma población, sumándose otras instituciones u organizaciones para generar desarrollo y solucionar los problemas territoriales.

Por su parte Cea, P. García, L. Nail, O. y Paredes, D., concluye que la gestión participativa garantiza superar los obstáculos de convivencia, llegando a mejorar las coordinaciones internas y unión de objetivos y compromisos de todos en las instituciones.

Sin embargo, Ramírez, N., Calderón, B., y Milián, M., menciona que aparte de la participación de los involucrados, la gestión articuladora y participativa, ayuda a mejorar diagnósticos de las propuestas, proponiendo mejores estrategias para lograr objetivos comunes, fomentando el desarrollo territorial y calidad de vida.

Así mismo, Assunção S., Acevedo, B., y Rodríguez, G., el diseño de acciones con todos los involucrados permite llevar a cabo la gestión participativa conllevando al desarrollo sostenible local.

Marín, F., Riquett, M., Pinto, M., Romero, S., y Paredes, A. La gestión participativa forma parte de la gestión política institucional, donde todos colaboran y comparten paradigmas para involucrarse en la solución de problemas comunes, desde diferentes espacios.

Sin embargo, Ramírez, N., Arana, N., y Guevara, M., menciona que la gestión participativa se mueve por los diferentes intereses y necesidades de los actores que convergen para el bienestar común de la comunidad. Para ello se sigue procesos de articulación y apoyo en todos los actores para lograr el desarrollo soñado. 
En este sentido, Lora, P. y Rocha, D. hacen énfasis a la interacción y el diálogo con diferentes conocimientos para realizar un diagnóstico articulado, donde englobe los problemas comunes y todos puedan ganar. Es decir, la gestión participativa es importante para la solución de objetivos comunes de todos los actores involucrados.

Cayotopa, C., concluye que la gestión participativa permite que todos los participantes sean actores del cambio, valorando los recursos disponibles y liderazgo de las instituciones como la municipalidad, sociedad civil y organismos privados.

Incluso, Garrido, M., y Hernández, A., concluyen que, la participación social, juega un papel importante desde la planificación, seguimiento y ejecución de acciones para lograr el desarrollo social anhelado.

La gestión participativa debe integrar procesos desde la planeación con miradas, horizontes, organización, ejecución y seguimiento a todo el proceso participativo, con la articulación de todos los actores involucrados, donde impera la participación activa, comunicación y compromisos para generar desarrollo. A su vez, todo el análisis de los autores, permitió corroborar el objetivo planteado en la investigación sobre Conocer la gestión participativa en el desarrollo de instituciones del estado, ya que todos los autores mencionan las características de la misma.

\section{CONCLUSIONES}

El análisis de todas las investigaciones, permitieron conocer la gestión participativa que influye en el desarrollo de las instituciones públicas, con diferentes puntos de vista, enfoque cualitativo y diseños descriptivos.

Se concluye que casi el $100 \%$ de las investigaciones, relacionan la influencia de la gestión participativa en el desarrollo de las instituciones públicas. Estas están basadas en políticas que tiene que ver con procesos desde la planificación, organización, dirección y control. Incluso, las instituciones inician el proceso desde la parte interna y los que deben fomentar la articulación externa con buenas políticas de comunicación para lograr la participación activa y compromisos de cada uno, con miras a generar desarrollo o llegar a objetivos comunes. A su vez, la gestión participativa no se aplica en la mayoría de las instituciones públicas, éstas trabajan de manera aislada como si fuera una diferente, no incluyen a los actores beneficiarios y otros involucrados en la elaboración de políticas y lineamientos que serán dirigidas para diferentes programas de la misma población. Para lograr la articulación de los diferentes actores depende mucho de la decisión política de los gobernantes o decisores de las instituciones, con vocación de servicio y miraras a generar desarrollo social. 


\section{REFERENCIAS}

Assunção S., Acevedo, B., y Rodríguez, G. (2016). La obra martiana en la gestión participativa ambiental para el desarrollo sostenible. Revista Universidad y Sociedad, vol.8 no.1 Cienfuegos Jan.-Apr. 2016, Cuba. Recuperado de: http://scielo.sld.cu/scielo.php?script=sci_arttext\&pid=S2218$36202016000100023 \& \operatorname{lng}=\mathrm{en} \& \ln \mathrm{l}=\mathrm{en} \#$ ?

Banco Central de Reserva del Perú - BCRP, (2017). Informe Económico y Social Región San Martín. Informe Económico, San Martín. Recuperado de: http://www.bcrp.gob.pe/docs/Proyeccion-Institucional/EncuentrosRegionales/2017/san-martin/ies-san-martin-2017.pdf

Bonnal, J., Cenerini, C., Ciparisse, J., y Rossi, M. (2013). El desarrollo territorial participativo y negociado (DTPN). La facilitación para la gobernanza territorial. Revista FAO, (4). Recuperado de: http://www.fao.org/docrep/019/mi207s/mi207s.pdf

Carta, J. y Pou, V. (2018). Productividad y eficiencia del sector público español. Observatorio Económico BBVA. Recuperado de: https://www.bbvaresearch.com/wpcontent/uploads/2018/07/Observatorio-productividad-sector-publico-1.pdf

Consorcio de Investigación Económica Social - CIES - (2016). Empleo informal y bienestar subjetivo en el Perú: Orientando las políticas públicas para un desarrollo social integral. Informe final. Recuperado de: https://www.cies.org.pe/sites/default/files/investigaciones/empleo_informal_y_bienest ar_subjetivo.pdf

Cayotopa, C. (2018). Modelo de gestión turística municipal integrada y participativa, para el fortalecimiento de la oferta turística cultural. Revista UCV - Hacer, Vol. 7 Núm. 3 (2018), Chiclayo. Recuperado de: http://revistas.ucv.edu.pe/index.php/UCVHACER/issue/view/194

Cea, P. Garcia, L. Nail, O. y Paredes, D. (2018). Gestión participativa en directivos para el abordaje de la convivencia escolar en instituciones educativas municipalizadas. Revista Iberoamericana de Educación. 2018;76(2):153-184. Chile. Recuperado de: https://doaj.org/article/a93190d83a8543c38849be5d5d0cede8?

Garrido, M., y Hernández, A. (2014). El patrimonio cultural: una propuesta de gestión participativa. Revista, Tejuelo, $\mathrm{n}^{\circ} 19$ (2014), págs. 62-75. España. Recuperado de: http://dehesa.unex.es/handle/10662/4509 
Lora, P. y Rocha, D. (2016). Promoción de la innovación social a través de la utilización de metodologías participativas en la gestión del conocimiento. Revista, Equidad \& Desarrollo, (25), 159-178. Bogotá. Recuperado de: http://dx.doi.org/10.19052/ed.3513

Marín, F., Riquett, M., Pinto, M., Romero, S., y Paredes, A. (2017). Gestión participativa y calidad educativa en el contexto del Plan de Mejoramiento Institucional en Escuelas Colombianas. Revista Opción, vol. 33, núm. 82, 2017, pp. 344-365 Universidad del Zulia. Maracaibo, Venezuela. Recuperado de: https://www.redalyc.org/pdf/310/31053180015.pdf

Ramírez, N., Calderón, B., y Milián, M. (2017). Gestión participativa en la elaboración de estrategias de revitalización urbana para el barrio histórico de San José, Puebla. Nova scientia vol.9 n.18. León, España. Recuperado de: http://www.scielo.org.mx/scielo.php?script=sci_arttext\&pid=S2007$07052017000100588 \& \operatorname{lng}=\mathrm{en} \& \ln \mathrm{l}=\mathrm{en} \#$ ?

Ramírez, N., Arana, N., y Guevara, M. (2019). "Participative management model for humanizing public spaces. Analco neighborhood, Puebla, Mexico". Revista Bitácora Urbano Territorial, 29 (1):43 - 52. Recuperado de: http://eds.a.ebscohost.com/eds/pdfviewer/pdfviewer?vid=0\&sid=ee4c9d1b-321d49d0-a0f9-ff24a668e79c\%40sessionmgr4008

Salgado, S., Flores, M., y Romero, M. (2017). Gestión participativa para mejorar las condiciones de accesibilidad Urbana: La Hacienda, Puebla. Revista Nova Scientia EISSN: 2007-0705 nova_scientia@delasalle.edu.mx Universidad De La Salle Bajío México. Recuperado de: http://www.redalyc.org/articulo.oa?id=203350918026 